\title{
Transient diabetes insipidus in a post-partum woman with pre-eclampsia associated with residual placental vasopressinase activity
}

\author{
Natassia Rodrigo and Samantha Hocking \\ Department of Endocrinology and Diabetes, Royal North Shore Hospital, Sydney, and University of Sydney, \\ Sydney, Australia
}

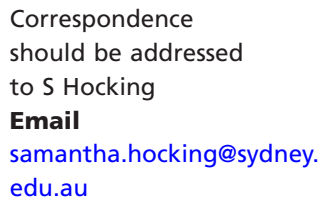

\section{Summary}

This case illustrates the exceedingly rare phenomenon of transient diabetes insipidus, in association with pre-eclampsia, occurring in the post-partum period following an in vitro fertilisation pregnancy, in an otherwise well 48-year-old lady. Diabetes insipidus can manifest during pregnancy, induced by increased vasopressinase activity secreted by placental trophoblasts and usually manifests in the third trimester. This presentation elucidates not only the intricate balance between the physiology of pregnancy and hormonal homeostasis, but also the importance of post-partum care as the physiological changes of pregnancy still hold pathological potential in the weeks immediately following delivery.

\section{Learning points:}

- Diabetes insipidus (DI) is a rare complication of pregnancy occurring in 1 in 30000 pregnancies.

- It is associated with excessive vasopressinase activity, secreted by placental trophoblasts, which increases the rate of degradation of anti-diuretic hormone.

- It is responsive to synthetic desmopressin 1-deanimo-8-D-arginine vasopressin as this form is not degraded by placental vasopressinase.

- Vasopressinase is proportional to placental weight, which is increased in pregnancies conceived with assisted reproductive techniques including in vitro fertilisation.

- Vasopressinase-induced DI is associated with pre-eclampsia.

\section{Background}

Pregnancy offers unique challenges to the diagnosis and management of endocrinopathies, as the physiological changes of pregnancy introduce diagnostic complexity into the interpretation of investigative results. Diabetes insipidus (DI) is a rare complication of pregnancy, occurring in 1 in 30000 pregnancies and can be difficult to recognise in late-pregnancy and the post-partum period as increased thirst, increased water intake and increased urine output are commonly reported. Furthermore, pregnancy is a state of water retention with increased maternal blood volume and an associated decrease in plasma osmolality. This case exemplifies these diagnostic intricacies in an exceptionally rare presentation of concomitant vasopressinase-induced DI and pre-eclampsia manifesting in the post-partum period.

\section{Case presentation}

A 48-year-old lady presented to the emergency department with a 3-day history of a progressively worsening, global 
headache. It was sudden in onset, initially intermittent in nature, now persistent. There were no aggravating or relieving factors. She denied any nausea or vomiting, visual disturbance, speech disturbance or focal weakness. She noted swelling of her legs, emergent over the last 3 days. Interestingly, she also complained of an insidious onset of polydipsia with increased oral intake to $5 \mathrm{~L}$ in the last $24 \mathrm{~h}$ prior to presentation. This was associated with polyuria and nocturia. Of note, she was 8 days postpartum, having delivered a $3.4 \mathrm{~kg}$ male infant by elective lower segment caesarean section (LSCS) at 38-week and 3-day gestation. The delivery was complicated by $500 \mathrm{~mL}$ blood loss. The current pregnancy was achieved through in vitro fertilisation (IVF) utilising a donor egg and was preceded by two prior pregnancies resulting in miscarriage. Gestational diabetes was diagnosed in the second trimester and managed by dietary modification alone, with no hypertension or proteinuria documented during the pregnancy. She had no other significant past medical or family history of disease and was not on any regular medications. She was an ex-smoker, with a 10 pack-year history. She denied any alcohol intake.

On examination, she was hypertensive to $183 / 87 \mathrm{mmHg}$, not tachycardic or tachypnoeic and was afebrile. Neurological examination revealed no focal deficits and reflexes that were globally brisk but not hyperreflexic. Cranial nerve examination was normal with no visual field defect. She was noted to have pitting oedema bilaterally to the knees and dry mucous membranes. Her cardiovascular examination was otherwise normal. Her abdominal examination was normal, and her surgical incision site appeared to be healing well.

\section{Investigation}

She underwent an urgent CT brain, which revealed an enlarged pituitary and queried lymphocytic hypophysitis or haemorrhage. Urine analysis demonstrated new-onset proteinuria with protein to creatinine ratio $110 \mathrm{mg} / \mathrm{mmol}$. Laboratory data were as follows: glucose $5.3 \mathrm{mmol} / \mathrm{L}$, sodium $146 \mathrm{mmol} / \mathrm{L}$, creatinine $150 \mu \mathrm{mol} / \mathrm{L}$, eGFR $35 \mathrm{~mL} /$ $\mathrm{min} / 1.73 \mathrm{~m}^{2}$ and urate $0.58 \mathrm{mmol} / \mathrm{L}(0.15-0.40)$. She had an isolated elevated alkaline phosphatase (ALP) of $143 \mathrm{U} / \mathrm{L}$ (20-105) with otherwise normal liver function tests. She had a normal platelet count of 258 (150-400). She was diagnosed with post-partum pre-eclampsia, however, did not meet the criteria for HELLP syndrome.

She remained polydipsic and polyuric, passing urine every $1-2 \mathrm{~h}$. Urine osmolality was $175 \mathrm{mmol} / \mathrm{kg}$ with a serum osmolality of $308 \mathrm{mmol} / \mathrm{kg}$. Pituitary MRI revealed a pituitary at the upper limit of normal considering her recent pregnancy, with no evidence of infarct or inflammation (Fig. 1). Anterior pituitary hormone profile was normal. She underwent a water deprivation test, which terminated after $4 \mathrm{~h}$, with sodium rising to $146 \mathrm{mmol} / \mathrm{L}$ with serum osmolality $302 \mathrm{mmol} / \mathrm{kg}$, urine osmolality $175 \mathrm{mmol} / \mathrm{kg}$ and anti-diuretic hormone (ADH) level $<0.8 \mathrm{ng} / \mathrm{L}$. She was diagnosed with DI. In the context of her pre-eclampsia, this was thought to be due to the residual action of placental vasopressinase.

\section{Treatment}

Following the diagnosis of pre-eclampsia, she was commenced on oxprenalol, resulting in the gradual resolution of her headache with normalisation of her blood pressure. Following confirmation of DI with the water deprivation test, she was commenced on desmopressin nasal spray, which resulted in decreased urine output.

\section{Outcome and follow-up}

On discharge creatinine had improved to $73 \mathrm{mmol} / \mathrm{L}$ and sodium was $142 \mathrm{mmol} / \mathrm{L}$. She returned to clinic 1 week post discharge. She had stopped taking desmopressin due to an upper respiratory tract infection. Post cessation, she did not have any nocturia and passed urine 5-6 times per day. She continued to drink to thirst. Sodium

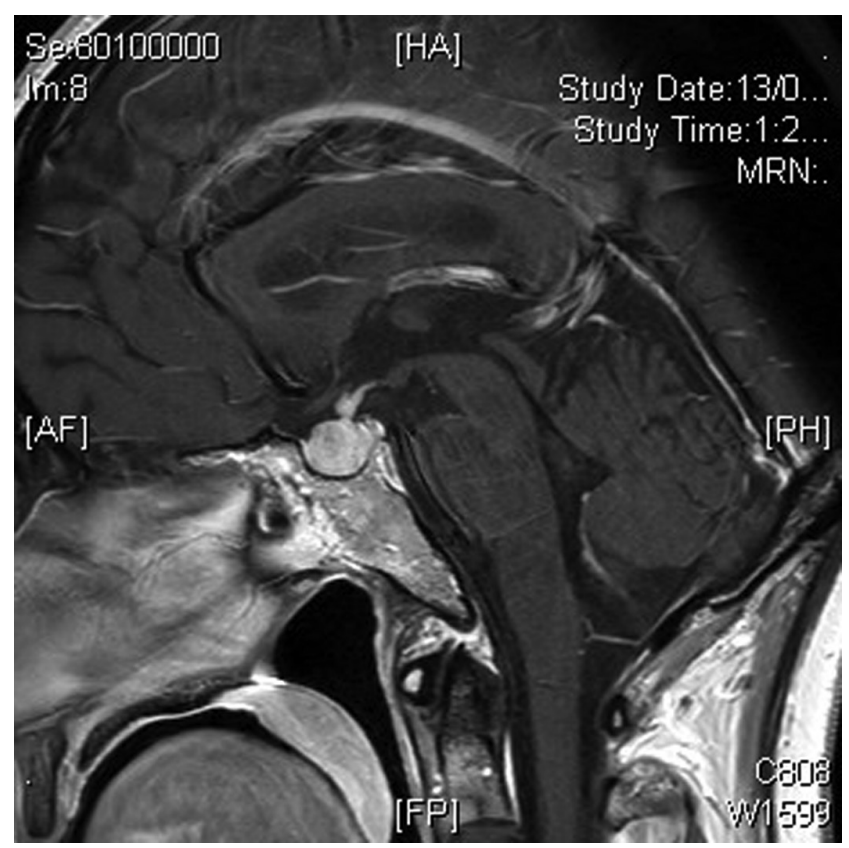

Figure 1

Pituitary MRI post-partum. 
Table 1 Trend in serum and urine markers over time.

\begin{tabular}{|c|c|c|}
\hline Date & Serum $\mathbf{N a}(\mathrm{mmol} / \mathrm{L})$ & Serum osmolality $(\mathrm{mmol} / \mathrm{kg})$ \\
\hline $12 / 3 / 2017$ & 140 & 308 \\
\hline $13 / 3 / 2017$ & 140 & 304 \\
\hline $14 / 3 / 2017$ & 140 & 304 \\
\hline $15 / 3 / 2017$ & 146 & 302 \\
\hline 16/3/2017 & 142 & 302 \\
\hline $17 / 3 / 2017$ & 139 & 302 \\
\hline 21/3/2017 & 142 & 303 \\
\hline 24/3/2017 & 142 & 295 \\
\hline $19 / 4 / 2017$ & 142 & 306 \\
\hline
\end{tabular}

\begin{tabular}{c}
\hline Urine osmolality $(\mathrm{mmol} / \mathrm{kg})$ \\
\hline 188 \\
175 \\
164 \\
175 \\
\\
\\
223 \\
267 \\
516 \\
\hline
\end{tabular}

\begin{tabular}{c}
\hline Urine $\mathbf{~ N a}(\mathrm{mmol} / \mathrm{L})$ \\
\hline 34
\end{tabular}

\begin{tabular}{c}
\hline ADH $(\mathrm{ng} / \mathrm{L})$ \\
\hline$<0.8$ \\
$<0.8$ \\
$<0.8$ \\
$<0.8$ \\
$<0.8$ \\
$<0.8$ \\
0.9 \\
7 \\
2.9
\end{tabular}

was $142 \mathrm{mmol} / \mathrm{L}$, serum osmolality $303 \mathrm{mmol} / \mathrm{kg}$, urine sodium $<20 \mathrm{mmol} / \mathrm{L}$, urine osmolality $223 \mathrm{mmol} / \mathrm{kg}$ and ADH $0.9 \mathrm{ng} / \mathrm{L}$. (Table 1)

By 1 month post discharge, her symptoms had resolved with resolution of both her pre-eclampsia and DI, such that she no longer required any medications.

\section{Discussion}

Gestational DI is a rare complication of pregnancy, occurring in 1 in 30000 pregnancies (1). It is characterised by hypotonic polyuria and polydipsia, manifesting as a transient phenomenon specific to the pregnant milieu, secondary to increased placental vasopressinase activity (1). It is associated with absent ADH levels, as demonstrated in our case (2). Placental vasopressinase, expressed in placental trophoblasts, degrades endogenous $\mathrm{ADH}$ but not 1-deanimo-8-D-arginine vasopressin (dDAVP), the synthetic form with a modified N-terminal, thereby allowing the resolution of symptoms with dDAVP administration (3).

Pregnancy denotes a change in maternal blood volume, osmoregulation and $\mathrm{ADH}$ secretion by the pituitary, whereby water retention increases and plasma osmolality decreases by $10 \mathrm{mmol} / \mathrm{kg}$ (4). There is a three-fold increase in the metabolic clearance of $\mathrm{ADH}$ in pregnancy, due to the excretion of vasopressinase from placental trophoblasts (4). Vasopressinase is a cystine aminopeptidase that inactivates $\mathrm{ADH}$ and oxytocin. It is produced from the seventh gestational week and concentrations rise coincidentally with the rise of trophoblastic mass, to peak in the third trimester. Following delivery, levels fall, becoming undetectable by $5-6$ weeks post-partum (5). This reflects the natural history of vasopressinase-induced transient DI, which typically has its onset in the third trimester and remits spontaneously by 4-6 weeks after delivery (6).

Post-partum presentations of isolated DI are exceedingly unusual (1). Post-partum DI has previously been described in relation to placental abruption, due to large volume release of placental vasopressinase into the blood stream (7). There are no reports of post-partum DI following placental manipulation through LSCS,or following post-partum haemorrhage with retained placenta.

Vasopressinase concentrations are commensurate with placental mass (5), reflecting the known increased incidence of vasopressinase-induced DI in multiple gestations, which are associated with increased trophoblastic mass (7). Another fascinating facet to our case is the associated IVF pregnancy. Pregnancies conceived with artificial reproductive technology (ART), including IVF and intra cytoplasmic sperm injection , are associated with larger placental weights, and a higher placental weight/birth weight ratio, independent of length of gestation at delivery or method of ART (8). Extrapolating from this, it can be conjectured that IVF pregnancies are at increased risk of transient DI through this mechanism. There are not data yet supporting this theory.

Post-partum pre-eclampsia is atypical but described (9). The pathophysiology of pre-eclampsia is yet to be fully elucidated, but placental dysfunction is emerging as critical to its pathophysiology. While commonly attributed risk factors include pre-existing hypertension, nulliparity, obesity and maternal age greater than 40 years (10), there is emerging recognition of the risk in IVF pregnancies. Egg donation is a highly successful form of ART, however, is emerging as a demonstrable risk for the development of pre-eclampsia with a recent meta-analysis denoting a odds ratio of 4.5 , compared to autologous egg IVF pregnancies (11). The postulated mechanism centres on discordant allogenicity of the foetus in relation to the mother (12). The use of a donor egg in this case, coupled with her advanced maternal age and nulliparous status, provide multiple risk factors for pre-eclampsia to develop.

Pre-eclamptic placental and end-organ dysfunction manifests in a myriad of ways, including maternal 
hypertension, proteinuria, thrombocytopenia, renal insufficiency, intrauterine growth restriction and neurological disturbance (13). Vasopressinase is metabolised in the liver, with vasopressinase-induced DI described in patients with eclampsia and pre-eclampsia, especially HELLP syndrome, as the associated liver dysfunction impairs clearance of vasopressinase (14). It has also been described in other forms of liver dysfunction complicating pregnancy, leading to deranged liver function, including hepatic steatosis (15). This is especially important in our patient as she had concomitant deranged liver function, providing a constellation of circumstances for gestational DI to manifest.

This case illuminates the diagnostic complexities of gestational DI, especially in the rare situation of postpartum emergence of disease. A high index of suspicion for vasopressinase-induced DI should thus be maintained in patients presenting with polyuria, especially in the presence of other risk factors such as pre-eclampsia and hepatic dysfunction, multiple gestation and IVF pregnancies.

\section{Declaration of interest}

The authors declare that there is no conflict of interest that could be perceived as prejudicing the impartiality of the research reported.

\section{Funding}

This research did not receive any specific grant from any funding agency in the public, commercial or not for profit sector.

\section{Patient consent}

Patient consent has been obtained.

\section{Author contribution statement}

Both authors were involved in patient care and in the writing of the manuscript.

\section{References}

1 Ananthakrishnan S. Diabetes insipidus in pregnancy: etiology, evaluation, and management. Endocrine Practice 200915 377-382. (https://doi.org/10.4158/EP09090.RA)
2 Barron WM, Cohen LH, Ulland LA, Lassiter WE, Fulghum EM, Emmanouel D, Robertson G \& Lindheimer MD. Transient vasopressin-resistant diabetes insipidus of pregnancy. New England Journal of Medicine 1984310 442-444. (https://doi.org/10.1056/ NEJM198402163100707)

3 Durr JA, Hoggard JG, Hunt JM \& Schrier RW. Diabetes insipidus in pregnancy associated with abnormally high circulating vasopressinase activity. New England Journal of Medicine 1987316 1070-1074. (https://doi.org/10.1056/ NEJM198704233161707)

4 Lindheimer MD, Barron WM \& Davison JM. Osmoregulation of thirst and vasopressin release in pregnancy. American Journal of Physiology 1989257 F159-F169.

5 Schrier RW. Systemic arterial vasodilation, vasopressin, and vasopressinase in pregnancy. Journal of the American Society of Nephrology 201021 570-572. (https://doi.org/10.1681/ ASN.2009060653)

6 Bellastella A, Bizzarro A, Colella C, Bellastella G, Sinisi AA \& De Bellis A. Subclinical diabetes insipidus. Best Practice and Research: Clinical Endocrinology and Metabolism 201226 471-483. (https://doi. org/10.1016/j.beem.2011.11.008)

7 Wallia A, Bizhanova A, Huang W, Goldsmith SL, Gossett DR \& Kopp P. Acute diabetes insipidus mediated by vasopressinase after placental abruption. Journal of Clinical Endocrinology and Metabolism 201398 881-886. (https://doi.org/10.1210/jc.2012-3548)

8 Haavaldsen C, Tanbo T \& Eskild A. Placental weight in singleton pregnancies with and without assisted reproductive technology: a population study of 536567 pregnancies. Human Reproduction 2012 27 576-582. (https://doi.org/10.1093/humrep/der428)

9 Stella CL \& Sibai BM. Preeclampsia: diagnosis and management of the atypical presentation. Journal of MaternalFetal and Neonatal Medicine 200619 381-386. (https://doi. org/10.1080/14767050600678337)

10 English FA, Kenny LC \& McCarthy FP. Risk factors and effective management of preeclampsia. Integrated Blood Pressure Control $2015 \mathbf{8}$ 7-12. (https://doi.org/10.2147/IBPC.S50641)

11 Schwarze JE, Borda P, Vásquez P, Ortega C, Villa S, Crosby JA \& Pommer R. Is the risk of preeclampsia higher in donor oocyte pregnancies? A systematic review and meta-analysis. JBRA Assisted Reproduction 201722 15-19. (https://doi.org/10.5935/15180557.20180001)

12 Blazquez A, García D, Rodríguez A, Vassena R, Figueras F \& Vernaeve $\mathrm{V}$. Is oocyte donation a risk factor for preeclampsia? A systematic review and meta-analysis. Journal of Assisted Reproduction and Genetics 201633 855-863. (https://doi.org/10.1007/s10815-0160701-9)

13 Sites CK, Wilson D, Barsky M, Bernson D, Bernstein IM, Boulet S \& Zhang Y. Embryo cryopreservation and preeclampsia risk. Fertility and Sterility 2017108 784-790. (https://doi.org/10.1016/j. fertnstert.2017.08.035)

14 Mor A, Fuchs Y, Zafra K, Haberman S \& Tal R. Acute presentation of gestational diabetes insipidus with pre-eclampsia complicated by cerebral vasoconstriction: a case report and review of the published work. Journal of Obstetrics and Gynaecology Research 201541 1269-1272. (https://doi.org/10.1111/jog.12694)

15 Jacques A, Durr M, Marshall M \& Lindheimer D., Diagnosis and management of diabetes insipidus during pregnancy. Endocrine Practice 19962 353-361. (https://doi.org/10.4158/EP.2.5.353) 